PACHECO, Yaritza Pérez; FERMÍN, Igor Alejandro. Impacto de las nuevas tecnologías en el arbitraje internacional. Revista Eletrônica Direito e Política, Programa de Pós-Graduação Stricto Sensu em Ciência Jurídica da UNIVALI, Itajaí, v.15, n.3, 30 quadrimestre de 2020. Disponível em: www.univali.br/direitoepolitica - ISSN 1980-7791

\title{
IMPACTO DE LAS NUEVAS TECNOLOGÍAS EN EL ARBITRAJE INTERNACIONAL ${ }^{1}$
}

\author{
IMPACTO DAS NOVAS TECNOLOGIAS NA ARBITRAGEM INTERNACIONAL
}

\section{Yaritza Pérez Pacheco ${ }^{2}$ Igor Alejandro Fermín ${ }^{3}$}

In a properly automated and educated world, then, machines may prove to be the true humanizing influence. It may be that machines will do the work that makes life possible and that human beings will do all the other things that make life pleasant and worthwhile.

Isaac Asimov (1990). Robot Visions.

\section{RESUMEN}

En este artículo pretende identificar cómo las tecnologías emergentes han redefinido la estructura tradicional del arbitraje internacional y determinar cómo la "inteligencia del dato" puede mejorar la toma de decisiones mejor informadas en esta materia. Partimos de la tesis según la cual la "inteligencia del dato", en el arbitraje internacional permitirá acelerar la toma de decisiones mejor informadas, dotando al arbitraje internacional de una verdadera eficacia, celeridad y reducción de costos; así como el acceso a un mayor número de justiciables. A partir del método deductivo con una exhaustiva investigación bibliográfica y de opiniones de expertos, se concluye que el uso ético de las tecnologías emergentes en el arbitraje internacional, tienen el potencial de renovar esta institución y posibilitar la toma de decisiones mejor informadas, lo que permitirá impulsar la automatización y gestión de casos en línea, con mayor eficiencia, precisión y transparencia.

\footnotetext{
1 Este artículo forma parte de la línea de la investigación "Derecho y Nuevas Tecnologías". Un primer avance en inglés será publicado en 2021, en el libro International Arbitration in the age of the Technological Revolution - Volume 2. Las opiniones expresadas en este documento son responsabilidad exclusiva de los autores y pueden no coincidir con las de las organizaciones con las cuales colaboran actualmente.

2 Estancia posdoctoral en el Programa Internacional Post-Doctorado en "Nuevas Tecnologías y Derecho" (2020-2021). Doctora en Derecho por la Universidad Nacional Autónoma de México (UNAM). Magister Scientiarum en Derecho Internacional Privado y Comparado y Abogada por la Universidad Central de Venezuela (UCV). Investigadora y docente en diversas universidades latinoamericanas. Ex directora de la Escuela de Derecho de la Facultad de Ciencias Jurídicas y Políticas de la UCV. Integrante del Sistema Nacional de Investigadores del CONACyT. Actualmente es la Subdirectora de Investigación del Centro de Investigaciones Judiciales de la Escuela Judicial del Estado de México, Toluca, México. Correo: yaritza.perez@pjedomex.gob.mx

${ }^{3}$ Experto en Transformación Digital y Asesoría de Valor, ex Director de Industria para Ciencias de la Salud y Educación Superior e Industria de Investigación en SAP Latinoamérica. Actualmente Especialista en Transformación Digital en el equipo de industrias de Google Cloud LATAM. Correo: igor.fermin@gmail.com
} 
PACHECO, Yaritza Pérez; FERMÍN, Igor Alejandro. Impacto de las nuevas tecnologías en el arbitraje internacional. Revista Eletrônica Direito e Política, Programa de Pós-Graduação Stricto Sensu em Ciência Jurídica da UNIVALI, Itajaí, v.15, n.3, 30 quadrimestre de 2020. Disponível em: www.univali.br/direitoepolitica - ISSN 1980-7791

PALABRAS CLAVE: Derecho y nuevas tecnologías; E-arbitraje; Servicios de arbitraje internacional; Justicia predictiva.

\section{RESUMO}

Neste artigo pretende-se identificar como as tecnologias emergentes têm redefinido a estrutura tradicional da arbitragem internacional e determinar como a "inteligência de dados" pode melhorar a tomada de decisões nestas matérias. Parte-se da tese segundo a qual a "inteligência de dados", na arbitragem internacional, permitirá acelerar a tomada de decisões informatizadas, dotando-a de uma verdadeira eficácia, celeridade, redução de custos e facilitação de acesso. A partir do método dedutivo com exaustiva investigação bibliográfica de opinião de especialistas, se conclui que o uso ético das tecnologias emergentes na arbitragem internacional tem o potencial de renovar o instituto e possibilitar a tomada de decisões informatizadas, o que permitirá impulsionar a automatização e gestão de casos como maior eficiência, precisão e transparência.

PALAVRAS-CHAVE: Direito e novas tecnologias; e-arbitragem; serviços de arbitragem internacional; justiça preditiva.

\section{INTRODUCCIÓN}

Big data, inteligencia artificial, blockchain, aprendizaje automático, análisis de datos y minería de texto son las nuevas tecnologías que están transformado significativamente las estructuras económicas y sociales $y$, con ello, nuestro estilo de vida, facilitando procesos autogestionables que hacen posible el acceso a la información de forma extendida. Lo anterior no ha logrado en la misma medida en la manera tradicional de resolver los problemas jurídicos, a pesar de que el conflicto es connatural al ser humano.

Somos testigos de una ola disruptiva de plataformas tecnológicas que adoptan nuevos modelos de usuarios altamente dependientes del ecosistema digital, lo que más temprano que tarde alcanzará a las estructuras tradicionales del Derecho. Si esta transformación será positiva o negativa aún no podemos predecirlo, pero es una transformación inevitable. No estamos afirmando la inexistencia del impacto tecnológico en la solución de controversias regladas por el Derecho, sino su lenta recepción, y hasta limitada, en comparación con otros sectores. 
PACHECO, Yaritza Pérez; FERMÍN, Igor Alejandro. Impacto de las nuevas tecnologías en el arbitraje internacional. Revista Eletrônica Direito e Política, Programa de Pós-Graduação Stricto Sensu em Ciência Jurídica da UNIVALI, Itajaí, v.15, n.3, 30 quadrimestre de 2020. Disponível em: www.univali.br/direitoepolitica - ISSN 1980-7791

En este contexto, el desarrollo del arbitraje internacional no está al margen de las nuevas tecnologías. Pero ¿cómo se proyecta el futuro del arbitraje ante estas nuevas tecnologías? ¿De qué manera afectará la forma en que se arbitran los casos? ¿Quiénes desempeñaran los roles principales en el arbitraje, los humanos o los robots? Son respuestas que no podemos proporcionar dado que se trata de acontecimientos en pleno desarrollo.

Si bien la resolución de controversias, tanto en sede jurisdiccional como en sede arbitral, es una batalla reglada, no cabe duda de que el éxito o el fracaso en un litigio está determinado por el conocimiento, la experiencia, la capacidad de persuasión y de convencimiento, esto es, por el "juego supremo de tácticas y estrategias" del equipo legal $y$, en este marco, la tecnología es un instrumento poderoso. 4

La tesis que sostenemos es que el arbitraje internacional se vale de las tecnologías emergentes solo como una herramienta para la comunicación, el almacenamiento y procesamiento de datos; pero los datos sin interpretación solo son datos sistematizados. En consecuencia, consideramos que la utilización de la "inteligencia del dato", es decir el aprovechamiento y el valor del dato en su contexto más amplio, permitirá a los actores del arbitraje internacional (instituciones arbitrales, partes y árbitros) acelerar la toma de decisiones mejor informadas, dotando al arbitraje internacional de una verdadera eficacia, celeridad y reducción de costos; lo que permitirá el acceso a un mayor número de justiciables, incrementándose así el alcance de este mecanismo alternativo de solución de controversias.

En los procedimientos de arbitraje suelen producirse grandes cantidades de información, para cuyo procesamiento se requiere invertir muchas horas de trabajo humano intelectual; pero la revisión de toda la documentación incorporada a los complejos procesos de arbitraje puede ser inacabada, debido al volumen, al tiempo que se dispone para su revisión, a las diferencias entre las distintas fuentes de datos, a la necesidad de conocimientos específicos para su almacenamiento, procesamiento e interpretación. Es aquí donde las tecnologías

\footnotetext{
${ }^{4}$ PEÑA, Daniel. "Nuevos escenarios del arbitraje: entre la Uberisación y la Inteligencia Artificial". Blog de Negocios (6 de abril, 2018). https://dernegocios.uexternado.edu.co/prospectiva/nuevosescenarios-del-arbitration-entre-la-uberisacion-y-la-inteligencia-artificial/
} 
PACHECO, Yaritza Pérez; FERMÍN, Igor Alejandro. Impacto de las nuevas tecnologías en el arbitraje internacional. Revista Eletrônica Direito e Política, Programa de Pós-Graduação Stricto Sensu em Ciência Jurídica da UNIVALI, Itajaí, v.15, n.3, 30 quadrimestre de 2020. Disponível em: www.univali.br/direitoepolitica - ISSN 1980-7791

emergentes proporcionan herramientas muy eficientes para la visualización y gestión documental.

Con este artículo nos proponemos dos objetivos: (i) identificar cómo el big data y las tecnologías emergentes, como la inteligencia artificial, el aprendizaje automático, los servicios de almacenamiento en la nube (cloud computing), han redefinido la estructura tradicional del arbitraje internacional; y (ii) determinar cómo el arbitraje internacional puede tomar ventaja de la "inteligencia del dato" en tiempos de convergencia tecnológica para la toma de decisiones mejor informadas.

En la actualidad, se pone a prueba cómo la ciencia de datos puede mejorar en gran medida la calidad y la eficiencia de los procesos legales, como el arbitraje internacional, desde el establecimiento de estrategias de litigio, pasando por el debate probatorio hasta la predicción de resultados. Las siguientes líneas pretenden dar a conocer el estado actual del conocimiento que se está produciendo en esta materia y aportar aproximaciones sobre su impacto real.

\section{IMPORTANCIA Y BENEFICIOS DE LA TECNOLOGÍA EN EL ARBITRAJE}

El arbitraje es un servicio legal que se promueve en los entornos nacionales e internacionales con características excepcionales frente a la jurisdiccional estatal. Se afirma que el arbitraje permite a los particulares resolver las controversias de manera eficiente, con árbitros especializados, procedimientos flexibles, expeditos y confidenciales, mediante un laudo arbitral de obligatorio cumplimiento para las partes.

Se trata de una justicia privada, financiada absolutamente por las partes; deslocalizada; sobre la base de un acuerdo de voluntades que impregna de flexibilidad y practicidad al arbitraje. Los árbitros tienen la investidura temporal de jueces y siguiendo las reglas procesales aplicables, así como los reglamentos institucionales o las reglas pactadas ad-hoc por las partes (reglas de arbitraje y legislación arbitral), toman decisiones definitivas sobre derechos e intereses en litigio. En este contexto, la tecnología puede lograr el supremo propósito de 
PACHECO, Yaritza Pérez; FERMÍN, Igor Alejandro. Impacto de las nuevas tecnologías en el arbitraje internacional. Revista Eletrônica Direito e Política, Programa de Pós-Graduação Stricto Sensu em Ciência Jurídica da UNIVALI, Itajaí, v.15, n.3, 30 quadrimestre de 2020. Disponível em: www.univali.br/direitoepolitica - ISSN 1980-7791

eficiencia, reducción de costos, seguridad jurídica, e incluso, decisiones más justas. ${ }^{5}$

La practicidad del arbitraje tiene mucho que ver con el uso de la tecnología, sus aplicaciones y alcances pueden resultar asombrosos, contrario a lo que sucede en los juicios que se llevan a cabo en las jurisdicciones estatales.

La actual revolución tecnológica afecta a lo jurídico, lo que incluye al arbitraje en dos aspectos fundamentales. Por un lado, las nuevas tecnologías contribuyen a convertir al arbitraje en uno de los sistemas de solución de controversias (junto con la mediación y la conciliación) más eficiente y, por tanto, ayuda a optimizar la administración de justicia privada. Por otro lado, la incorporación de la tecnología, no solo como una herramienta para la comunicación, el almacenamiento y procesamiento de datos, como una herramienta que permite la visualización e interpretación del dato, supone un desafío para el Derecho, pero para el arbitraje internacional puede implicar transformaciones importantes que contribuyan a la democratización de este mecanismo, ${ }^{6}$ en aras de revertir los cuestionamientos sobre corrupción y falta de transparencia en estos procedimientos.

En definitiva, el arbitraje debe sacar el máximo provecho al uso de las tecnologías, porque se complementan recíprocamente, ambos buscan la eficiencia y la rapidez. Se aspira que las instituciones arbitrales incorporen el uso de la tecnología durante toda la vida del arbitraje, impulsando las productividad y colaboración, es decir, desde el acuerdo de arbitraje, pasando por el desarrollo del procedimiento, hasta la conclusión y ejecución del laudo arbitral que al efecto se dicte. Veamos algunos de los beneficios que se pueden potenciar con la incorporación de las nuevas tecnologías al arbitraje internacional.

\subsection{Inclusión del uso de la tecnología en el convenio arbitral}

\footnotetext{
${ }^{5}$ PEÑA, Daniel. "Nuevos escenarios del arbitraje: entre la Uberisación y la Inteligencia Artificial".

6 ROGERS, James \& BUCKLE, Matthew. "The future of arbitration in the world of Big Data". International Arbitration Report, No 9 October, 2017, p. 13.
} 
PACHECO, Yaritza Pérez; FERMÍN, Igor Alejandro. Impacto de las nuevas tecnologías en el arbitraje internacional. Revista Eletrônica Direito e Política, Programa de Pós-Graduação Stricto Sensu em Ciência Jurídica da UNIVALI, Itajaí, v.15, n.3, 30 quadrimestre de 2020. Disponível em: www.univali.br/direitoepolitica - ISSN 1980-7791

El arbitraje encuentra su origen y fundamento en el acuerdo de voluntades entre las partes, a través del cual establecen cómo se llevará a cabo el procedimiento en caso de que se active la cláusula arbitral que sustrae las controversias objeto del convenio de la jurisdicción estatal.

En estos casos se recomienda señalar el uso de la tecnología en el acuerdo de arbitraje, pero sin especificar cuál de ellas, por cuanto los desarrollos en esta materia suelen ser acelerados. Además, esta inclusión en la cláusula arbitral tiene por finalidad el desarrollo eficiente y económico del procedimiento de arbitraje. $^{7}$

Entre los beneficios más evidentes podemos mencionar la facilidad de transmisión y almacenamiento de datos; la reducción de costos asociados a los traslados y logística de las audiencias presenciales a través del uso de la videoconferencia; pero en un sitial muy importantes y representativos en estos tiempos de ataques cibernéticos, se encuentran la seguridad, la protección de datos y la privacidad de las partes.

\subsection{Activación de la cláusula de resolución de controversias}

Si bien, las partes pueden decidir cuáles son las reglas aplicables al procedimiento de arbitraje, en la práctica existen instituciones arbitrales que brindan servicios para la administración de este procedimiento y ponen a disposición de las partes sus reglamentos.

Estos centros están comenzando a adoptar prácticas más eficientes, incorporando la tecnología a los procesos de gestión administrativa y procesal desde el inicio del arbitraje. Es así como comienzan a aparecer plataformas automatizadas para la presentación de solicitudes de arbitraje, lo que debería llegar a traducirse en reducción de tiempos y costos monetarios, así como menor impacto ambiental al reducir al mínimo el uso del papel. Como sabemos, en los arbitrajes internacionales se manejan grandes volúmenes de información que

7 CORONADO AGUILAR, Guillermo. "La tecnología dentro del arbitraje". COEM.MX (2017, 16 de octubre). https://coem.mx/la-tecnologia-dentro-del-arbitration/ 
PACHECO, Yaritza Pérez; FERMÍN, Igor Alejandro. Impacto de las nuevas tecnologías en el arbitraje internacional. Revista Eletrônica Direito e Política, Programa de Pós-Graduação Stricto Sensu em Ciência Jurídica da UNIVALI, Itajaí, v.15, n.3, 30 quadrimestre de 2020. Disponível em: www.univali.br/direitoepolitica - ISSN 1980-7791

suelen ser reproducidas no solo para la contraparte, sino también para cada uno de los miembros del tribunal arbitral y la entidad administradora del mismo.

Como puede evidenciarse, la tecnología puede ser un gran aliado para agilizar, dotar de eficiencia y sustentabilidad al procedimiento de arbitraje en esta primera etapa.

\subsection{Desarrollo del procedimiento de arbitraje}

Lo ideal es adoptar prácticas que promuevan, una vez iniciado el arbitraje y el tribunal arbitral este constituido, que todas las partes involucradas determinen cuáles serán las tecnologías de la información que les permitirán un procedimiento más eficiente. El momento más oportuno para determinarlo es en el acta de misión o en la primera orden procesal; si las partes no alcanzan ningún acuerdo al respecto, los árbitros pueden guiarlas en cuanto al uso de las tecnologías que consideren más apropiadas.

Así, las comunicaciones entre las partes y el tribunal arbitral desde hace mucho tiempo se realizan vía correo electrónico; el intercambio de pruebas documentales a través de memorias USB o flash drives y servicios de almacenamiento en la nube a través de plataformas de colaboración como Google Drive $\AA$, Wetransfer $\AA$ o Dropbox; también el uso de videoconferencias para la celebración de audiencias, declaración de testigos y presentación de peritos expertos con herramientas como Skype $(\AA)$ o FaceTime $\AA$, para que sin la presencia física en un tribunal arbitral se desarrolle el procedimiento arbitral, lo que reduce a cero los gastos de traslado, sin sacrificar los principios de oralidad e inmediación. ${ }^{8}$

Esto ya es una realidad en algunas instituciones de arbitraje. Por ejemplo: el artículo 22 (2) de las Reglamento de Arbitraje de la Cámara de Comercio Internacional del año 2017 establece que: "Con el fin de asegurar la conducción efectiva del caso, el tribunal arbitral, previa consulta a las partes, podrá adoptar las medidas procesales que considere apropiadas, siempre que éstas no vulneren

8 TEMBOURY, Miguel. "Arbitraje y nuevas tecnologías, condenados a entenderse". Diario Jurídico (19 de octubre, 2010). https://www.diariojuridico.com/arbitration-y-nuevas-tecnologiascondenados-a-entenderse/ 
PACHECO, Yaritza Pérez; FERMÍN, Igor Alejandro. Impacto de las nuevas tecnologías en el arbitraje internacional. Revista Eletrônica Direito e Política, Programa de Pós-Graduação Stricto Sensu em Ciência Jurídica da UNIVALI, Itajaí, v.15, n.3, 30 quadrimestre de 2020. Disponível em: www.univali.br/direitoepolitica - ISSN 1980-7791

ningún acuerdo de las partes". Otro ejemplo interesante lo encontramos en las Reglas de la IBA sobre obtención de pruebas en el arbitraje internacional, aprobadas el 29 de mayo de 2010, las cuales prevén que "Cada testigo deberá comparecer en persona salvo que en casos excepcionales el Tribunal Arbitral permita el uso de videoconferencia o de una tecnología similar" (artículo 8.1).

La situación tras la pandemia ocasionada por el Covid-19 (SARS-CoV-2) aceleró la implementación de herramientas tecnológicas, en aras de evitar retrasos en el desarrollo de los procedimientos de arbitraje. ${ }^{9}$ Como afirma Iordache:

Technology is becoming a second 'discipline', alongside law, animating arbitration proceedings. This is not new or COVID19-related, but, certainly, not being able to meet in person for months had removed much of the resistance to technology in ADR proceedings. ${ }^{10}$

\subsection{Reducción de costos}

Tal vez una de las ventajas más cuestionadas del uso de las tecnologías en el arbitraje sea el impacto en los costos. Con la promesa de agilizar todas las fases del arbitraje —desde la selección de árbitros hasta el cálculo de daño_, han surgido en el mercado software de productividad y colaboración, servicios de almacenamiento en la nube y plataformas de desarrollo de aplicaciones que facilitan la innovación a gran escala.

Si bien, estas nuevas tecnologías pueden resultar muy útiles para el mejor desarrollo del procedimiento de arbitraje, es poco probable, en el corto plazo,

\footnotetext{
9 En Perú, el Poder Judicial autorizó que los abogados y litigantes puedan entrevistarse con los jueces y administradores de los módulos básicos de justicia y módulos corporativos de las cortes superiores del país, mediante la aplicación "Google Hangouts Meet". Esto se aplicará mientras dure el estado de emergencia ocasionado por el Covid-19 (SARS-CoV-2) e incluso durante el período de reactivación progresiva de las labores en las dependencias judiciales (La Ley, 30 de Abril de 2020, https://laley.pe/art/9648/autorizan-que-abogados-se-entrevisten-con-los-jueces-mediante-googlehangouts-meet). También en Brasil, se presentan algunas iniciativas para continuar funcionando en estos tiempos de aislamiento social, la Escuela Superior de la Magistratura de Alagoas (Esmal) imparte clases virtuales y en vivo, a través de la plataforma Google Meet (CGJ, 10 de julio de 2020, https://cgj.tjal.jus.br/?pag=verNoticia\&not=17068).

10 "La tecnología se está convirtiendo en una segunda 'disciplina', junto con el Derecho, animando los procedimientos de arbitraje. Esto no es nuevo ni está relacionado con COVID-19, pero, ciertamente, no poder reunirse en persona durante meses ha eliminado gran parte de la resistencia a la tecnología en los procedimientos de ADR" (traducción libre). IORDACHE, Adrian. Simpler: Building a Robot and How to Think of ADR Tech. Kluwer Arbitration Blog (16 de junio, 2020). http://arbitrationblog.kluwerarbitration.com/2020/06/16/simpler-building-a-robot-and-how-tothink-of-adr-tech/?doing_wp_cron=1597018670.8120489120483398437500\#respond
} 
PACHECO, Yaritza Pérez; FERMÍN, Igor Alejandro. Impacto de las nuevas tecnologías en el arbitraje internacional. Revista Eletrônica Direito e Política, Programa de Pós-Graduação Stricto Sensu em Ciência Jurídica da UNIVALI, Itajaí, v.15, n.3, 30 quadrimestre de 2020. Disponível em: www.univali.br/direitoepolitica - ISSN 1980-7791

que impacte en los costos de los servicios legales que ofrecen las instituciones o centros de arbitraje. ${ }^{11}$ La implementación de herramientas tecnológicas de inteligencia artificial, por ejemplo, podrían resultar prohibitivamente costosas para la mayoría de los despachos de abogados y pequeños centros de arbitraje, por los altos costos de inversión que se requieren, por lo menos al inicio, con una tasa de retorno que deberá evaluarse en cada caso.

Sin embargo, en el actual escenario, caracterizado por una velocidad exponencial en el desarrollo e innovación de soluciones tecnológicas, se muestran algunas facilidades para la adopción de modelos tecnológicos a bajo costo, los cuales permitirán aumentar el tamaño de las organizaciones, como por ejemplo el procesamiento de inteligencia artificial en la nube. La idea central es innovar desde los actuales flujos de trabajo.

\section{ARBITRAJE Y DISRUPCIÓN TECNOLÓGICA}

Al arbitraje se le atribuyen muchas ventajas, pero sus detractores lo acusan de ser costoso, ineficiente y elitista. Si bien, por una parte, las instituciones arbitrales se mercadean ofreciendo ventajas en la relación eficiencia-tiemposcostos; por otra parte, las críticas se enfilan hacia el procedimiento de arbitraje, la conducta de las partes, los centros de arbitraje y a los mismos árbitros. Pero no nos centremos en los aspectos negativos, sino en los retos significativos que afronta el arbitraje ante la disrupción tecnológica.

El arbitraje y las nuevas tecnologías comparten una relación de mutuo beneficio. Por un lado, las nuevas tecnologías tienen el potencial para contribuir a un mayor nivel de eficiencia, reducir costos, promover la expansión del arbitraje en nuevos segmentos de mercados y mejorar los resultados para los clientes. ${ }^{12}$ Como afirma Lee, pasamos de "the age of expertise the age of data". En efecto:

Today, successful AI [artificial intelligence] algorithms need three things: big data, computing power, and the work of strong - but not necessarily elite - AI algorithm engineers.

\footnotetext{
11 TURCHI, Megan. "The future of international arbitration may not be AI". ThinKset (7 de marzo, 2019). https://thinksetmag.com/issue-7/ai-may-not-be-the-future-of-international-arbitration

12 VANNIEUWENHUYSE, Gauthier. "Arbitration and new technologies: mutual benefits". Journal of International Arbitration, Vol. 35, N 1, 2018.
} 
PACHECO, Yaritza Pérez; FERMÍN, Igor Alejandro. Impacto de las nuevas tecnologías en el arbitraje internacional. Revista Eletrônica Direito e Política, Programa de Pós-Graduação Stricto Sensu em Ciência Jurídica da UNIVALI, Itajaí, v.15, n.3, 30 quadrimestre de 2020. Disponível em: www.univali.br/direitoepolitica - ISSN 1980-7791

Bringing the power of deep learning to bear on new problems requires all there, but in this age of implementation data is core. ${ }^{13}$

En este apartado, planteamos algunas respuestas a la pregunta sobre ¿cómo el big data y la convergencia tecnológica han redefinido la estructura tradicional del arbitraje internacional? Veamos algunas iniciativas que se están presentando en este sector:

\subsection{Aumento en la oferta $y$ demanda de servicios de arbitraje internacional}

Las relaciones entre oferta y demanda son modificadas de manera esencial por la utilización eficiente de la información; pero con la implementación de plataformas tecnológicas para la administración y gestión procesal del arbitraje es posible atraer un número mayor de controversias hacia las instituciones que se dedican a este tipo de servicios jurídicos. ${ }^{14}$

El arbitraje es una realidad en las controversias que se suscitan en el eCommerce, entre proveedores y consumidores; se destacan en este sector eBay y PayPal empresas que ofrecen resolución de disputas originadas por el intercambio comercial en línea, a través de sistemas totalmente automatizados conocidos como resolución de disputas en línea conocidos en inglés por sus siglas ODR (online dispute resolution), que consisten en desarrollar de forma "online" procedimientos de resolución de disputas que salven las barreras geográficas entre las partes enfrentadas, con o sin intervención de profesionales (humanos) que las asistan.

\footnotetext{
13 "...la era de la experiencia a la era de los datos. Hoy en día, los algoritmos de inteligencia artificial exitosos necesitan tres cosas: big data, poder de cómputo y el trabajo de ingenieros de algoritmos de inteligencia artificial fuertes, pero no necesariamente de élite. Llevar el poder del aprendizaje profundo para resolver nuevos problemas requiere que todo esté ahí, pero en esta era de implementación, los datos son fundamentales" (traducción libre). LEE, Kai-Fu. AI Superpowers: China, Silicon Valley, and the New World Order. New York: Houghton Mifflin Harcourt, 2018, p. 14.

${ }^{14}$ PEÑA, Daniel. "Nuevos escenarios del arbitraje: entre la Uberisación y la Inteligencia Artificial".
} 
PACHECO, Yaritza Pérez; FERMÍN, Igor Alejandro. Impacto de las nuevas tecnologías en el arbitraje internacional. Revista Eletrônica Direito e Política, Programa de Pós-Graduação Stricto Sensu em Ciência Jurídica da UNIVALI, Itajaí, v.15, n.3, 30 quadrimestre de 2020. Disponível em: www.univali.br/direitoepolitica - ISSN 1980-7791

\subsection{Digitalización de los procedimientos de arbitraje}

El arbitraje internacional usualmente es un servicio administrado por centros o instituciones privadas que prestan una labor de apoyo a las partes y a los árbitros durante todas las etapas del procedimiento. Para crear escenarios más eficientes y dinámicos es necesario que estos centros incorporen plataformas que acerquen a los usuarios entre sí, que permitan determinar la arquitectura del sistema de información y por ende sus funcionalidades.

En este sentido, un primer paso lo constituye la digitalización del proceso arbitral, desde la recepción de la solicitud, el nombramiento de árbitros, peritos y seguimiento de todas las etapas a través de plataformas automatizadas con accesos cifrados para todas las partes involucradas, lo que en definitiva se traducirá en mayor eficiencia, al mismo tiempo liberará al árbitro de muchas de las cargas del manejo de casos. ${ }^{15}$

Algunas instituciones de arbitraje han dado un paso adelante en este sentido; por ejemplo, la Corte de Arbitraje de la Cámara de Comercio e Industria de Madrid, la cual cuenta con un sistema para la tramitación de arbitrajes on-line (TAO-OAM), disponible desde el mes de julio de 2010. Se presenta como un sistema que permite a las partes en un procedimiento de arbitraje acceder a su expediente las 24 horas del día y los siete días de la semana, desde cualquier parte del mundo, ya sea para consulta del expediente o llevar a cabo algún trámite en el procedimiento de arbitraje. Con lo cual se favorece la comunicación instantánea entre las partes, los árbitros y la Corte, eliminando los tiempos de traslado y dotando así a los procedimientos de mayor agilidad y celeridad. Otro ejemplo, es el arbitraje online del Centro de Arbitraje de la Cámara de Comercio de Lima, el cual permite presentar las solicitudes de arbitraje exclusivamente a través de una plataforma electrónica dispuesta para tales fines denominada "Mesa de Partes Virtual", la cual está operativa desde el 4 de mayo de 2020.

15 KLIMOV, Yaroslav \& PANOV, Andrey. "Procedural innovations in arbitration". International Arbitration Report, No 9 October 2017, pp. 32-33. 
PACHECO, Yaritza Pérez; FERMÍN, Igor Alejandro. Impacto de las nuevas tecnologías en el arbitraje internacional. Revista Eletrônica Direito e Política, Programa de Pós-Graduação Stricto Sensu em Ciência Jurídica da UNIVALI, Itajaí, v.15, n.3, 30 quadrimestre de 2020. Disponível em: www.univali.br/direitoepolitica - ISSN 1980-7791

\subsection{Ejecución de los laudos arbitrales}

Las tecnologías emergentes como smart contracts (contrato inteligente) y blockchain pueden mejorar considerablemente los tiempos de ejecución de los laudos arbitrales, pero también en una etapa anterior y posterior, esto es, tanto para la materialización del acuerdo de las partes de someter sus controversias a arbitraje como para la ejecución del laudo arbitral que pone fin al arbitraje.

Nos referimos a la posibilidad de celebrar un contrato en el cual se incorpore una cláusula de arbitraje en formato smart contracts, de tal manera que sea suficiente programar las condiciones contractuales en la plataforma digital para que el contrato pueda ejecutarse automáticamente; así el código se activa por sí mismo y genera efectos patrimoniales. ${ }^{16}$ Esto es, a través de un protocolo informático se verifica el cumplimiento de las condiciones contractuales y se ejecuta el contrato de manera automática, siendo el mismo sistema el que garantiza y controla el cumplimiento de las obligaciones.

El blockchain es una tecnología con un registro inmutable de información o transacciones entre personas que no requiere de un intermediario para funcionar. Aun cuando se dio a conocer como una herramienta para transacciones financieras y en concreto se asocia a las criptomonedas, su alcance es superior en la actualidad: smart contracts, registros públicos descentralizados, transacciones descentralizadas en los mercados de energía eléctrica y, el tema que nos interesa en este estudio, el arbitraje internacional como uno de los medios o mecanismos que integra los sistemas alternativos de resolución de conflictos, conocidos en inglés por su acrónimo ADR (alternative dispute resolution).

El blockchain puede tener diversas aplicaciones en el arbitraje, como lo destaca Duarte, ${ }^{17}$ pero queremos destacar su aplicación en la ejecución automática del laudo arbitral final, lo cual es posible cuando las partes involucradas han

\footnotetext{
${ }^{16}$ GONZÁLEZ ARANGO, Julio César \& CRUZ MANTILLA, Santiago. 'Blockchain' y sus desafíos en materia de resolución de disputas". Legis Ámbito Jurídico (11 octubre, 2019). https://www.ambitojuridico.com/noticias/especiales/mercantil-propiedad-intelectual-yarbitraje/blockchain-y-sus-desafios-en.

${ }^{17}$ DUARTE, Mauricio. "El futuro del arbitraje radica en el uso del Blockchain. CIAR Global" (16 abril, 2019). https://ciarglobal.com/el-futuro-del-arbitraje-radica-en-el-uso-del-blockchain/
} 
PACHECO, Yaritza Pérez; FERMÍN, Igor Alejandro. Impacto de las nuevas tecnologías en el arbitraje internacional. Revista Eletrônica Direito e Política, Programa de Pós-Graduação Stricto Sensu em Ciência Jurídica da UNIVALI, Itajaí, v.15, n.3, 30 quadrimestre de 2020. Disponível em: www.univali.br/direitoepolitica - ISSN 1980-7791

acordado una adjudicación por medio de activos digitales (utilizando mecanismos similares a la ejecución automática para los contratos inteligentes), con la implementación de protocolos informáticos adecuados.

\section{BIG DATA, INTELIGENCIA ARTIFICIAL Y ARBITRAJE}

El procesamiento de macrodatos, también llamados datos masivos, datos a gran escala o big data es una tendencia que está permeando en todos los sectores, incluido el arbitraje, la cual consiste en recopilar, procesar, almacenar y analizar grandes volúmenes de información con el propósito de orientar la toma de decisiones de cualquier individuo, organización o institución pública o privada, a partir de algoritmos complejos. El big data no puede separarse de los avances en la inteligencia artificial, ya que implica la posibilidad de interpretar grandes volúmenes de información generando respuestas y soluciones basadas en preferencias y sistemas automatizados. ${ }^{18}$

En este sentido, la inteligencia artificial es tal vez la tecnología con mayor potencial de irrupción en el arbitraje internacional, dada su capacidad para replicar y aumentar las habilidades cognitivas humanas, automatizar tareas simples, pero que consumen mucho tiempo, y procesar cantidades masivas de datos. $^{19}$ En un principio, la inteligencia artificial se limitaba a buscar palabras claves, ahora puede analizar y extraer información relevante de material escrito, correos electrónicos y conversaciones de voz.

Si bien, a la fecha, el uso básico de la inteligencia artificial en los despachos jurídicos está dirigido a administrar documentos en grandes cantidades que antes eran revisados y verificados por abogados junior, empleando para ello mucho tiempo y dinero. En el futuro inmediato, las potencialidades que brinda la inteligencia artificial, a través del procesamiento del lenguaje natural, con capacidad para analizar decenas de miles o cientos de miles de documentos relevantes para un litigio se considerará una herramienta indispensable para cualquier abogado. Como afirman Rhim \& Park: "The types of Legal Tech

\footnotetext{
18 BECERRA, Jairo. Derecho y big data. Universidad Católica de Colombia. Bogotá, 2018, p. 141,143-144.

19 TURCHI, Megan. "The future of international arbitration may not be $\mathrm{AI}^{\prime}$.
} 
PACHECO, Yaritza Pérez; FERMÍN, Igor Alejandro. Impacto de las nuevas tecnologías en el arbitraje internacional. Revista Eletrônica Direito e Política, Programa de Pós-Graduação Stricto Sensu em Ciência Jurídica da UNIVALI, Itajaí, v.15, n.3, 30 quadrimestre de 2020. Disponível em: www.univali.br/direitoepolitica - ISSN 1980-7791

industry are as diverse as the Word of lawyers. In the end, all the services with the nickname of AI lawyer can be considered as one of Legal Techs". ${ }^{20}$

En efecto, en los últimos años se han dado a conocer varias herramientas de "análisis de datos", diseñadas para ayudar a los abogados a predecir el resultado de sus disputas ante los tribunales estatales. Una de las primeras fue "Lex Machina", en 2010, como resultado de un proyecto de interés público de la Facultad de Derecho de Stanford. Actualmente, es un producto de LexisNexis company que ofrece predecir el comportamiento de tribunales, jueces, abogados y partes con Legal Analytics. Cada vez es más frecuente evidenciar como el mundo de la tecnología se está ocupando de las aplicaciones que pueden tener las nuevas tecnologías en el Derecho.

Hoy contamos con varios productos tecnológicos aplicables al Derecho y al arbitraje. Cada uno parece adoptar ángulos ligeramente diferentes en su mensaje de marketing; por ejemplo, algunos se centran en los antecedentes del caso con miras a predecir los resultados probables. Otros incorporan un enfoque basado en la utilidad económica de la solución, usando la "teoría de juegos" en lugar de la "teoría de la decisión" para predecir las probables consecuencias económicas para una de las partes de resolver, o no resolver, una disputa en un determinado momento. ${ }^{21}$

Sin temor a equivocarnos, el empleo de la inteligencia artificial para gestionar los datos que se producen en cada controversia sometida a arbitraje permitirá hacer más eficiente el proceso arbitral.

Finalmente, tratemos de determinar cómo el arbitraje internacional puede tomar ventaja de la "inteligencia del dato" en tiempos de convergencia tecnológica, para la toma de decisiones mejor informadas.

\footnotetext{
20 "Los tipos de industria de tecnología legal son tan diversos como la palabra de los abogados. Al final, todos los servicios con el sobrenombre de abogado de IA pueden considerarse como uno de los Técnicos Jurídicos" (traducción libre). RHIM, Young-Yik \& PARK, Kyung Bae. "The applicability of artificial intelligence in international law". Journal of East Asia \& International Law, Vol. 12, $\mathrm{N}^{\circ} 1$, 2019, p. 14. http://dx.doi.org/10.14330/jeail.2019.12.1.01

${ }^{21}$ MAXWELL, Karen. "Computer says no: data analytics in arbitration". Practical Law Arbitration (9 de febrero, 2018). http://arbitrationblog.practicallaw.com/computer-says-no-data-analytics-inarbitration/
} 
PACHECO, Yaritza Pérez; FERMÍN, Igor Alejandro. Impacto de las nuevas tecnologías en el arbitraje internacional. Revista Eletrônica Direito e Política, Programa de Pós-Graduação Stricto Sensu em Ciência Jurídica da UNIVALI, Itajaí, v.15, n.3, 30 quadrimestre de 2020. Disponível em: www.univali.br/direitoepolitica - ISSN 1980-7791

\subsection{Redacción de cláusula de arbitraje}

En ocasiones, las cláusulas arbitrales incluidas en los contratos son tomadas de otros contratos $y$, en el mejor de los casos, de cláusulas modelo suministradas por las instituciones de arbitraje. Si se utiliza la inteligencia artificial en esta tarea, se podría lograr cláusula de arbitraje que tengan en consideración las particularidades del negocio jurídico, los derechos y obligaciones pactados, la comparación con otros contratos y arbitrajes similares, así como muchos elementos que auxiliarían a los clientes y abogados a reducir los errores, identificar los puntos ciegos y garantizar la protección de sus intereses. ${ }^{22}$

Hoy en día los grandes avances tecnológicos en la inteligencia artificial favorecen añadir diferentes funciones como visión, idioma, conversación y datos que ofrecen ventajas en los diferentes niveles de las organizaciones pudiendo llevar la idea a la práctica de forma rápida y eficiente.

\subsection{Simplificación de tareas administrativas}

La propuesta de valor fundamental de inteligencia artificial radica en su capacidad para agilizar las tareas administrativas a cargo de árbitros y abogados, quienes podrían delegar estas labores a las máquinas y concentrarse en los aspectos del proceso arbitral que requieren mayor cantidad de raciocinio humano: evaluar los hechos, construir argumentos y deliberar para determinar los resultados del caso. ${ }^{23}$

Las herramientas digitales son un ejemplo de gestión de documentos, para lo cual es necesario contar con toda la documentación del procedimiento de arbitraje en formato digital y realizar audiencias a través de plataformas de videoconferencias, entre otros recursos tecnológicos.

Las herramientas de aprendizaje automático (machine learning) utilizadas en este contexto podrían aprovecharse al máximo para procesar la información documental permitiendo un análisis más eficiente.

22 TURCHI, Megan. "The future of international arbitration may not be AI".

23 TURCHI, Megan. "The future of international arbitration may not be AI". 
PACHECO, Yaritza Pérez; FERMÍN, Igor Alejandro. Impacto de las nuevas tecnologías en el arbitraje internacional. Revista Eletrônica Direito e Política, Programa de Pós-Graduação Stricto Sensu em Ciência Jurídica da UNIVALI, Itajaí, v.15, n.3, 30 quadrimestre de 2020. Disponível em: www.univali.br/direitoepolitica - ISSN 1980-7791

\subsection{Cuantificación de daños}

El 9 de octubre de 2018, con motivo de la Conferencia Anual de la IBA en Roma, el Grupo de Trabajo sobre Daños de ICCA-ASIL reunió a profesionales y árbitros para desarrollar un enfoque coherente en esta materia. Se trata de un prototipo de aplicación web diseñado para brindar a los árbitros y practicantes un enfoque consistente para determinar los daños. ${ }^{24}$ Es importante advertir que la aplicación no hace cálculos; la idea es proporcionar un mapa, para ayudar a asegurarse de que los árbitros no se pierdan los problemas o se encuentren sin los datos que necesitan para continuar.

\subsection{Acceso a los datos}

Como ya sabemos, una de las ventajas del arbitraje es su confidencialidad, con lo cual cualquier herramienta de la inteligencia artificial requerirá que estén disponibles los datos adecuados para su procesamiento. Pero esta ventaja puede ser el principal obstáculo para el uso del análisis de datos en el arbitraje. Se requiere que los datos estén disponibles públicamente para que la herramienta tecnológica este en capacidad de analizarlos, de forma rápida y precisa. Pero la confidencialidad del arbitraje impide que contemos con datos completos sobre los árbitros y sus laudos arbitrales.

Sin embargo, parece no haber barreras infranqueables para la tecnología, desde hace un par de años está disponible en el mercado Dispute Resolution Data, LLC (DRD), la cual se promociona como "la primera y única base de datos global relacionada con disposiciones de mediación y arbitraje comercial internacional". De hecho, DRD fue galardonado como Mejor Innovación en 2017, por Global Arbitraje Review.

Obsérvese que estas plataformas tecnológicas buscan recopilar la mayor cantidad de datos posibles sobre un mismo caso. Esto no está exento de riesgos y desafíos, como los que plantea Bhargava:

The growing ubiquity of data and the myriad ways it can be collected raise big questions about how to make it truly

\footnotetext{
${ }^{24}$ TURCHI, Megan. "The future of international arbitration may not be AI".
} 
PACHECO, Yaritza Pérez; FERMÍN, Igor Alejandro. Impacto de las nuevas tecnologías en el arbitraje internacional. Revista Eletrônica Direito e Política, Programa de Pós-Graduação Stricto Sensu em Ciência Jurídica da UNIVALI, Itajaí, v.15, n.3, 30 quadrimestre de 2020. Disponível em: www.univali.br/direitoepolitica - ISSN 1980-7791

useful, who owns the data, and who should stand to profit from it $[\ldots]$ The unsurprising result of our data-reverent culture is that everyone is focused on finding new and better ways to amass more of it. Not only do companies constantly collect "big data" through the platforms they own, but our devices are generating perpetual streams of "small data" too. ${ }^{25}$

En este contexto, DRD asegura que, para preservar la confidencialidad de los arbitrajes sobre los cuales se realiza el análisis de datos (y de los cuales depende el éxito de las instituciones de arbitraje), el sitio web no solicitará ni publicará ninguna información de las instituciones de arbitraje con respecto a la identidad de las partes, los nombres del caso, defensores, árbitros o mediadores, ni nombres de instituciones asociadas con sus datos. En efecto, expresa en su página web que: "Los datos redactados se presentan en conjunto por tipo de caso y región geográfica evitando preocupaciones tradicionales de confidencialidad".

La inteligencia artificial también podría ayudar a las partes en una disputa a elegir al árbitro más idóneo, examinando los antecedentes de miles de candidatos en casos similares.

\subsection{Justicia predictiva}

Mientras que la tecnología es capaz de ayudar a los humanos en trabajos mecánicos y repetitivos, existe cierto consenso entre los expertos sobre la baja probabilidad de que puedan ser reemplazados en la toma de decisiones. Nosotros no estamos muy seguros de ello. En la práctica, no debemos perder de vista, lo que consideran Christensen \& Raynor:

Few technologies or business ideas are intrinsically sustaining or disruptive in character. Rather, their disruptive

\footnotetext{
25 "La creciente ubicuidad de los datos y las innumerables formas en que se pueden recopilar plantean grandes preguntas sobre cómo hacer que sean realmente útiles, quién es el propietario de los datos y quién debería beneficiarse de ellos [...] El resultado nada sorprendente de nuestra reverencia a los datos La cultura es que todos están enfocados en encontrar nuevas y mejores formas de acumular más. Las empresas no solo recopilan constantemente 'macrodatos' a través de las plataformas que poseen, sino que nuestros dispositivos también generan flujos perpetuos de 'pequeños datos' (traducción libre). BHARGAVA, Rohit. Non-Obvious Megatrends: How to See What Others Miss and Predict the Future (Non-Obvious Trends Series). Ideapress Publishing, 2020, p. 149.
} 
PACHECO, Yaritza Pérez; FERMÍN, Igor Alejandro. Impacto de las nuevas tecnologías en el arbitraje internacional. Revista Eletrônica Direito e Política, Programa de Pós-Graduação Stricto Sensu em Ciência Jurídica da UNIVALI, Itajaí, v.15, n.3, 30 quadrimestre de 2020. Disponível em: www.univali.br/direitoepolitica - ISSN 1980-7791

impact must be molded into strategy as manager shape the idea into plan and then implement it.

Successful new-growth builders know - either intuitively or explicitly - that disruptive strategies greatly increase the odds of competitive success.

Because disruption happens whether we want it or not. ${ }^{26}$

Para algunos, el análisis de datos para la elección de los árbitros es una manera de presidir resultados en el arbitraje, por cuanto se basan en las decisiones de los árbitros en casos anteriores. Sin embargo, esto no resuelve las duras críticas que se presentan sobre la designación de ciertos árbitros para ciertos tipos de disputas, en desmedro de la diversidad y la transparencia que exigen estos nombramientos. ${ }^{27}$

Es interesante la reflexión que hace Nieva-Fenoll sobre la posibilidad de que las máquinas puedan dictar sentencias desplazando así a los jueces de carne y hueso, lo cual es perfectamente aplicable al laudo arbitral y a los árbitros, al considerar que "la motivación [de las decisiones] es una labor que refleja la convicción de un juez y, por tanto, es eminentemente humana". Si bien, la inteligencia artificial "puede ayudar en algunos aspectos de la elaboración de la resolución, pero no en todos ellos, no siempre y ni siquiera de manera completa, pero tampoco despreciable". ${ }^{28}$

Las herramientas de "justicia predictiva" utilizan la inteligencia artificial para analizar situaciones que se presentan antes, durante y después del arbitraje, con el fin de obtener estadísticas sobre cómo se decidirá probabilísticamente en el próximo caso. ${ }^{29}$

\footnotetext{
26 "Pocas tecnologías o ideas comerciales tienen un carácter intrínsecamente sustentador o disruptivo. Más bien, su impacto disruptivo debe moldearse en una estrategia a medida que el gerente da forma a la idea en un plan y luego lo implementa.

Los constructores exitosos de nuevos crecimientos saben, ya sea de manera intuitiva o explícita, que las estrategias disruptivas aumentan en gran medida las probabilidades de éxito competitivo.

Porque la disrupción ocurre lo queramos o no" (traducción libre). CHRISTENSEN, Clayton M. \& RAYNOR, Michael E. The Innovator's Solution: Creating and Sustaining Successful Growth. Harvard Business Review Press, 2013, p. 23.

27 MAXWELL, Karen. "Computer says no: data analytics in arbitration". Practical Law Arbitration.

${ }^{28}$ NIEVA-FENOLL, Jordi. Inteligencia artificial y proceso judicial. Marcial Pons, 2018, p. 99.

29 MAXWELL, Winston; GOUIFFÈS, Laurent y VANNIEUWENHUYSE, Gauthier. "The future of arbitration: New technologies are making a big impact - and AI robots may take on 'human'
} 
PACHECO, Yaritza Pérez; FERMÍN, Igor Alejandro. Impacto de las nuevas tecnologías en el arbitraje internacional. Revista Eletrônica Direito e Política, Programa de Pós-Graduação Stricto Sensu em Ciência Jurídica da UNIVALI, Itajaí, v.15, n.3, 30 quadrimestre de 2020. Disponível em: www.univali.br/direitoepolitica - ISSN 1980-7791

\section{CONSIDERACIONES FINALES}

A corto plazo, no parece posible que la decisión arbitral se adopte en su integridad por una "máquina", pero el uso de herramientas automatizadas puede disminuir el error humano y producir laudos ejecutables bajo la revisión de un sistema de expertos legales (legal expert system).

El big data y la inteligencia artificial son aplicaciones tecnológicas que posibilitan la toma de decisiones mejor informadas en el ámbito del Derecho, en el cual se incluye el arbitraje internacional.

El uso ético de la artificial inteligencia en el análisis de las diferentes etapas del procedimiento de arbitraje permitirá fortalecer la institución desde la transparencia, por cuanto impulsará la automatización y gestión de casos en línea, con mayor eficiencia y precisión en la aplicación de los sistemas legales.

Como toda invención humana la inteligencia artificial puede incurrir en errores, si los datos suministrados o el algoritmo no son bien supervisados, una aplicación de inteligencia artificial podría sacar conclusiones falsas o cometer otros errores similares a los que se pretenden erradicar con su implementación. Por lo que no debe perderse de vista que la inteligencia artificial es de naturaleza asistencial, ayuda con los individuos a procesar la información que se requiere para adoptar decisiones mejor informadas.

Las tecnologías emergentes tienen el potencial de renovar los procedimientos de arbitraje.

\section{REFERENCIAS DE FUENTES CITADAS}

ASIMOV, Isaac. Robot Visions (First Ed) [E-book]. New York, N.Y., U.S.A.: Penguin Books, 1990.

BECERRA, Jairo. Derecho y big data. Universidad Católica de Colombia. Bogotá, 2018.

BHARGAVA, Rohit. Non-Obvious Megatrends: How to See What Others Miss and Predict the Future (Non-Obvious Trends Series). Ideapress Publishing, 2020.

roles" (21 de febrero, 2018). https://www.hoganlovells.com/en/publications/the-future-ofarbitration-ai-robots-may-take-on-human-roles 
PACHECO, Yaritza Pérez; FERMÍN, Igor Alejandro. Impacto de las nuevas tecnologías en el arbitraje internacional. Revista Eletrônica Direito e Política, Programa de Pós-Graduação Stricto Sensu em Ciência Jurídica da UNIVALI, Itajaí, v.15, n.3, 30 quadrimestre de 2020. Disponível em: www.univali.br/direitoepolitica - ISSN 1980-7791

CHRISTENSEN, Clayton M. \& RAYNOR, Michael E. The Innovator's Solution: Creating and Sustaining Successful Growth. Harvard Business Review Press, 2013.

CORONADO Aguilar, Guillermo. "La tecnología dentro del arbitraje". COEM.MX (16 de octubre, 2017). https://coem.mx/la-tecnologia-dentro-delarbitration/

Diamandis, Peter H. \& Kotler, Steven. The Future Is Faster Than You Think: How Converging Technologies Are Transforming Business, Industries, and Our Lives (Exponential Technology Series). New York: Simon \& Schuster, 2020.

DUARTE, Mauricio. "El futuro del arbitraje radica en el uso del Blockchain. CIAR Global" (16 de abril, 2019). https://ciarglobal.com/el-futuro-del-arbitrajeradica-en-el-uso-del-blockchain/

GONZÁLEZ ARANGO, Julio César \& CRUZ MANTILLA, Santiago. 'Blockchain' y sus desafíos en materia de resolución de disputas". Legis Ámbito Jurídico (11 octubre, 2019).

https://www.ambitojuridico.com/noticias/especiales/mercantil-propiedadintelectual-y-arbitraje/blockchain-y-sus-desafios-en

ICCA. Report on The Task Force Damages Breakfast at the IBA Annual Conference in Rome, 2018. https://www.arbitration-icca.org/projects/ICCAASIL-Task-Force-on-Damages.html

IORDACHE, Adrian. Simpler: Building a Robot and How to Think of ADR Tech. Kluwer Arbitration Blog (16 de junio, 2020). http://arbitrationblog.kluwerarbitration.com/2020/06/16/simpler-building-arobot-and-how-to-think-of-adrtech/?doing_wp_cron=1597018670.8120489120483398437500\# respond

KLIMOV, Yaroslav \& PANOV, Andrey. "Procedural innovations in arbitration". International arbitration report, No 9 October 2017, pp. 32-34.

LEE, Kai-Fu. AI Superpowers: China, Silicon Valley, and the New World Order. New York: Houghton Mifflin Harcourt, 2018.

MAXWELL, Winston, GOUIFFÈS, Laurent y VANNIEUWENHUYSE, Gauthier. "The future of arbitration: New technologies are making a big impact - and AI robots may take on 'human' roles" (21 de febrero, 2018). https://www.hoganlovells.com/en/publications/the-future-of-arbitration-airobots-may-take-on-human-roles

MAXWELL, Karen. "Computer says no: data analytics in arbitration". Practical Law Arbitration (9 de febrero, 2018). http://arbitrationblog.practicallaw.com/computer-says-no-data-analytics-inarbitration/

NIEVA-FENOLL, Jordi. Inteligencia artificial y proceso judicial. Marcial Pons, 2018. 
PACHECO, Yaritza Pérez; FERMÍN, Igor Alejandro. Impacto de las nuevas tecnologías en el arbitraje internacional. Revista Eletrônica Direito e Política, Programa de Pós-Graduação Stricto Sensu em Ciência Jurídica da UNIVALI, Itajaí, v.15, n.3, 30 quadrimestre de 2020. Disponível em: www.univali.br/direitoepolitica - ISSN 1980-7791

PEÑA, Daniel. Nuevos escenarios del arbitraje: entre la Uberisacion y la Inteligencia Artificial. Blog de Negocios (6 de abril, 2018). https://dernegocios.uexternado.edu.co/prospectiva/nuevos-escenarios-delarbitration-entre-la-uberisacion-y-la-inteligencia-artificial/

RHIM, Young-Yik \& PARK, Kyung Bae. "The applicability of artificial intelligence in international law". Journal of East Asia \& International Law, Vol. 12, No $1, \quad 2019$, pp. 7-30. Disponible. http://dx.doi.org/10.14330/jeail.2019.12.1.01. Consultado en: 30/08/2020.

ROGERS, James \& BUCKLE, Matthew. "The future of arbitration in the world of Big Data". International Arbitration Report, No 9 October, 2017, pp. 12-14.

TEMBOURY, Miguel. "Arbitraje y nuevas tecnologías, condenados a entenderse". Diario Jurídico (19 de octubre, 2010). https://www.diariojuridico.com/arbitration-y-nuevas-tecnologias-condenados-aentenderse/

TURCHI, Megan. "The future of international arbitration may not be $\mathbf{A I}$ ". ThinKset (2019, 7 march). https://thinksetmag.com/issue-7/ai-may-not-be-thefuture-of-international-arbitration

VANNIEUWENHUYSE, Gauthier. "Arbitration and new technologies: mutual benefits". Journal of International Arbitration, Vol. 35, No 1, 2018, pp. 119-129. 